\title{
Development of a Large-Scale Route to Glecaprevir: Synthesis of the Macrocycle via Intramolecular Etherification
}

Jeffrey M. Kallemeyn, Kenneth M. Engstrom, Matthew J. Pelc, Kirill A. Lukin, Westin H. Morrill, Haojuan Wei, Timothy B. Towne, Jeremy Henle, Nandkishor K. Nere, Dennie S. Welch, Shashank Shekhar, Matthew M. Ravn, Gang Zhao, Michael G. Fickes, Chen Ding, John C. Vinci, James Marren, and Russell D. Cink*

Process Research \& Development, and Analytical Research \& Development, AbbVie Inc., 1401

Sheridan Road, North Chicago, IL, 60064

* Email: russell.cink@abbvie.com 


\section{Table of Contents}

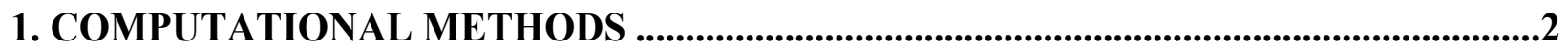

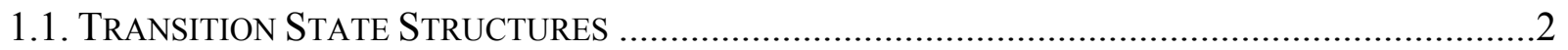

1.1.1. Pro-trans Carbonate Transition State Structure ...................................................2

1.1.2. Pro-cis Carbonate Transition State Structure ........................................................... 4

1.1.3. Pro-trans Epoxide Transition State Structure ...................................................... 6

1.1.4. Pro-cis Epoxide Transition State Structure ........................................................... 7

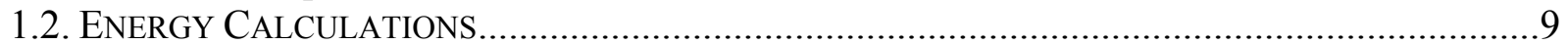

1.3. DisCusSiON OF NEGATIVE HYPERCONJUGATION IN THE TS AS A STABILIZING FACTOR........9

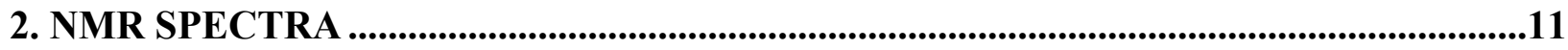

3. SINGLE CRYSTAL X-RAY STRUCTURE DETAILS ...................................................19

3.1. Macrocycle 3 P-Xylene Solvate Single Crystal Structure .................................19

3.2. MACrocyCle 3 Toluene Solvate Single Crystal Structure ..................................19

3.3. Macrocycle 3 Acetonitrile Solvate Single Crystal Structure ..........................20 


\section{Computational Methods}

All DFT calculations were performed using Jaguar version 10.5, release 13 as provided in the Schrodinger suite (version 2019-3) ${ }^{1,2}$ Transition states were optimized at the B3LYP-D3/6$311 \mathrm{G}^{* *++}$ level of theory in the gas phase. Single point energies were calculated at the B3LYPD3/6-311 G**++/SM8 level of theory, with THF solvation for the carbonate substrate and benzene solvation for the epoxide substrate. All single point energies had thermal corrections applied calculated at the B3LYP-D3/6-311G**++ level of theory on gas phase transition state structures.

\subsection{Transition State Structures}

\subsubsection{Pro-trans Carbonate Transition State Structure}
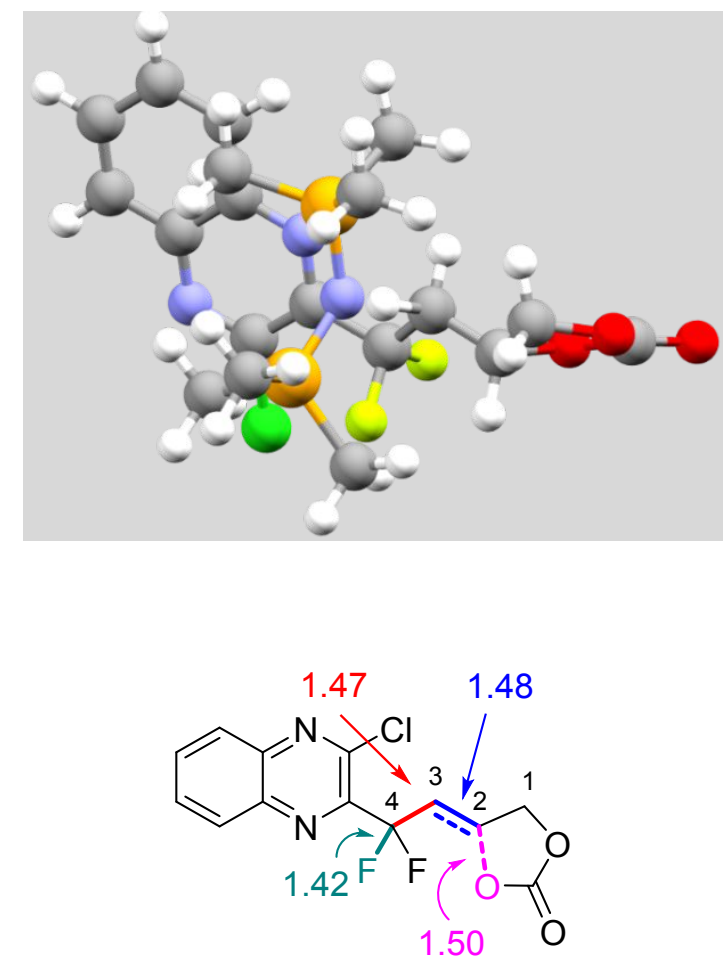

carbonate-TS trans

$\Delta \Delta \mathrm{G}^{\ddagger}{ }_{\mathrm{rel}}=0 \mathrm{kcal} / \mathrm{mol}$

$\begin{array}{lrrr}\text { F } & 2.1259 & 1.7710 & 0.2609 \\ \mathrm{C} & 0.9555 & 1.0063 & 0.3646 \\ \mathrm{C} & 0.6291 & 0.7930 & 1.7804 \\ \mathrm{C} & 0.5250 & 2.0175 & 2.6059 \\ \mathrm{O} & -0.7729 & 2.7657 & 2.4865\end{array}$

${ }^{1}$ Bochevarov, A.D.; Harder, E.; Hughes, T.F.; Greenwood, J.R.; Braden, D.A.; Philipp, D.M.; Rinaldo, D.; Halls, M.D.; Zhang, J.; Friesner, R.A., "Jaguar: A high-performance quantum chemistry software program with strengths in life and materials sciences," Int. J. Quantum Chem., 2013, 113(18), 2110-2142

${ }^{2}$ Schrodinger Release 2019-3: Jaguar, Schrodinger, LLC, New York, NY, 2019. 


\begin{tabular}{|c|c|c|c|}
\hline C & -1.1419 & 3.2222 & 3.6866 \\
\hline $\mathrm{O}$ & -0.3283 & 2.7434 & 4.6703 \\
\hline $\mathrm{C}$ & 0.5422 & 1.7388 & 4.1082 \\
\hline $\mathrm{O}$ & -2.0658 & 3.9582 & 3.9023 \\
\hline $\mathrm{F}$ & 0.0140 & 1.7783 & -0.3693 \\
\hline $\mathrm{C}$ & 1.1629 & -0.2752 & -0.4464 \\
\hline $\mathrm{C}$ & 1.7392 & -0.2945 & -1.7729 \\
\hline $\mathrm{Cl}$ & 2.1129 & 1.1917 & -2.6284 \\
\hline $\mathrm{N}$ & 1.9890 & -1.3900 & -2.4190 \\
\hline $\mathrm{C}$ & 1.6609 & -2.5701 & -1.8229 \\
\hline $\mathrm{C}$ & 1.9299 & -3.7958 & -2.4738 \\
\hline $\mathrm{C}$ & 1.5622 & -4.9787 & -1.8735 \\
\hline $\mathrm{C}$ & 0.9150 & -4.9805 & -0.6150 \\
\hline $\mathrm{C}$ & 0.6484 & -3.7999 & 0.0386 \\
\hline $\mathrm{C}$ & 1.0266 & -2.57 & -0.5512 \\
\hline $\mathrm{N}$ & 0.8058 & -1.4033 & 0.1080 \\
\hline $\mathrm{H}$ & -0.2217 & 0.1266 & 1.9030 \\
\hline $\mathrm{H}$ & 1.6945 & 0.0479 & 2.3597 \\
\hline $\mathrm{H}$ & 1.2899 & 2.7488 & 2.3375 \\
\hline $\mathrm{H}$ & 0.1357 & 0.7513 & 4.3 \\
\hline $\mathrm{H}$ & 1.5338 & 1.8304 & 4.5449 \\
\hline $\mathrm{H}$ & 2.4222 & -3.7693 & -3.4 \\
\hline $\mathrm{H}$ & 1.7702 & -5.9219 & -2.3669 \\
\hline $\mathrm{H}$ & 0.6402 & -5.9241 & -0.1576 \\
\hline $\mathrm{H}$ & 0.1760 & -3.7794 & 1.0119 \\
\hline $\mathrm{N}$ & 2.6504 & -0.6240 & 2.9778 \\
\hline $\mathrm{Si}$ & 4.2259 & -0.0875 & 2.5932 \\
\hline $\mathrm{Si}$ & 2.1734 & -2.0939 & 3.6938 \\
\hline $\mathrm{C}$ & 4.7230 & -0.5264 & 0.8107 \\
\hline $\mathrm{C}$ & 4.3594 & 1.7968 & 2.7566 \\
\hline $\mathrm{C}$ & 5.5748 & -0.8192 & 3.7234 \\
\hline $\mathrm{C}$ & 0.2862 & -2.2893 & 3.6181 \\
\hline $\mathrm{C}$ & 2.9308 & -3.6406 & 2.8721 \\
\hline $\mathrm{C}$ & 2.6422 & -2.2259 & 5.5350 \\
\hline $\mathrm{H}$ & 4.6717 & -1.6090 & \\
\hline $\mathrm{H}$ & 4.0439 & -0.0480 & 0.0996 \\
\hline $\mathrm{H}$ & 5.7405 & -0.1959 & 0.5713 \\
\hline $\mathrm{H}$ & 4.1245 & 2.1161 & 3.7776 \\
\hline $\mathrm{H}$ & 5.3706 & 2.1455 & 2.5177 \\
\hline $\mathrm{H}$ & 3.6652 & 2.2910 & 2.0732 \\
\hline $\mathrm{H}$ & 5.6219 & -1.9106 & 3.6516 \\
\hline $\mathrm{H}$ & 6.5604 & -0.4257 & 3.4480 \\
\hline $\mathrm{H}$ & 5.3898 & -0.5618 & 4.7711 \\
\hline $\mathrm{H}$ & -0.0115 & -3.2957 & 3.934 \\
\hline $\mathrm{H}$ & -0.2156 & -1.5766 & 4.280 \\
\hline $\mathrm{H}$ & -0.0811 & -2.1140 & 2.604 \\
\hline
\end{tabular}




$\begin{array}{llll}\mathrm{H} & 2.5195 & -4.5658 & 3.2941 \\ \mathrm{H} & 2.7441 & -3.6475 & 1.7951 \\ \mathrm{H} & 4.0159 & -3.6651 & 3.0178 \\ \mathrm{H} & 3.7287 & -2.2366 & 5.6660 \\ \mathrm{H} & 2.2476 & -1.3704 & 6.0928 \\ \mathrm{H} & 2.2411 & -3.1405 & 5.9880\end{array}$

1.1.2. Pro-cis Carbonate Transition State Structure
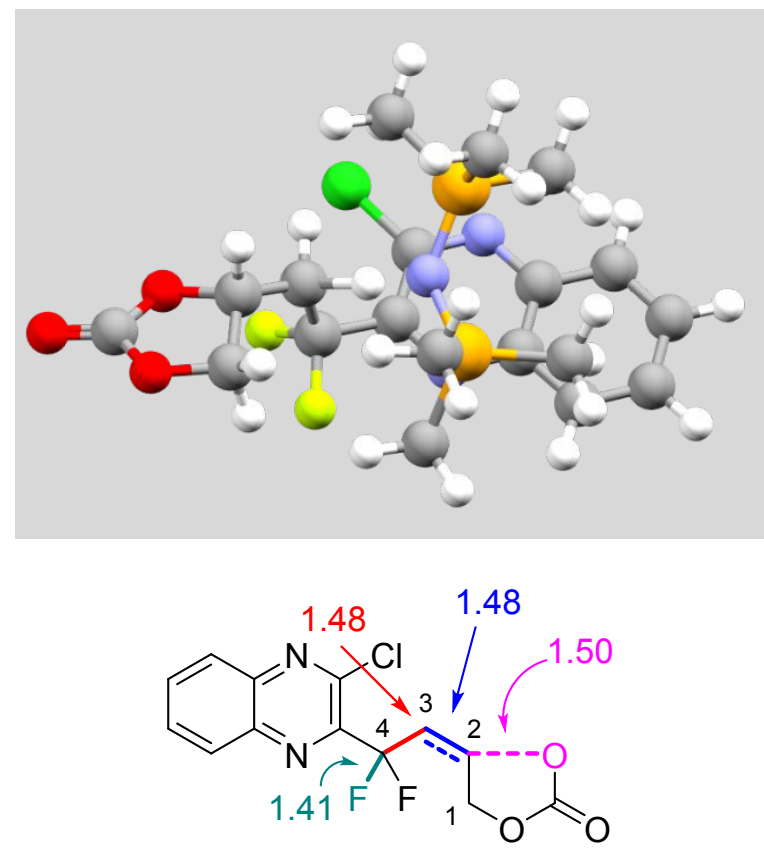

carbonate-TS cis $\Delta \Delta \mathrm{G}_{\mathrm{rel}}^{\ddagger}=4.4 \mathrm{kcal} / \mathrm{mol}$

$\begin{array}{lrrr}\text { F } & 0.2655 & 1.9365 & -0.3290 \\ \mathrm{C} & 1.0191 & 0.9915 & 0.4041 \\ \mathrm{C} & 2.3646 & 1.4337 & 0.8349 \\ \mathrm{C} & 2.5353 & 2.4962 & 1.8561 \\ \mathrm{O} & 1.9351 & 3.8312 & 1.5209 \\ \mathrm{C} & 1.6387 & 4.4710 & 2.6574 \\ \mathrm{O} & 1.7609 & 3.6415 & 3.7328 \\ \mathrm{C} & 1.9619 & 2.2912 & 3.2550 \\ \mathrm{O} & 1.3130 & 5.6222 & 2.7502 \\ \mathrm{~F} & 0.1932 & 0.6995 & 1.4730 \\ \mathrm{C} & 1.1486 & -0.2754 & -0.4537 \\ \mathrm{C} & 1.7295 & -0.2971 & -1.7760 \\ \mathrm{Cl} & 2.1347 & 1.1944 & -2.6197 \\ \mathrm{~N} & 1.9696 & -1.3894 & -2.4274 \\ \mathrm{C} & 1.6614 & -2.5711 & -1.8226 \\ \mathrm{C} & 1.9538 & -3.7967 & -2.4614 \\ \mathrm{C} & 1.6436 & -4.9812 & -1.8328\end{array}$




$\begin{array}{lrrr}\mathrm{C} & 1.0307 & -4.9835 & -0.5573 \\ \mathrm{C} & 0.7341 & -3.8009 & 0.0807 \\ \mathrm{C} & 1.0506 & -2.5707 & -0.5405 \\ \mathrm{~N} & 0.8029 & -1.4026 & 0.1070 \\ \mathrm{H} & 3.0779 & 0.3127 & 1.3219 \\ \mathrm{H} & 2.9445 & 1.6797 & -0.0518 \\ \mathrm{H} & 3.6081 & 2.7004 & 1.9406 \\ \mathrm{H} & 1.0032 & 1.7777 & 3.2477 \\ \mathrm{H} & 2.6596 & 1.7831 & 3.9185 \\ \mathrm{H} & 2.4333 & -3.7694 & -3.4323 \\ \mathrm{H} & 1.8779 & -5.9247 & -2.3128 \\ \mathrm{H} & 0.8070 & -5.9283 & -0.0750 \\ \mathrm{H} & 0.2849 & -3.7744 & 1.0659 \\ \mathrm{~N} & 3.8491 & -0.7096 & 1.6915 \\ \mathrm{Si} & 5.2559 & -0.9004 & 0.7425 \\ \mathrm{Si} & 3.3878 & -1.6729 & 3.0256 \\ \mathrm{C} & 5.5767 & 0.6447 & -0.3123 \\ \mathrm{C} & 6.8343 & -1.1994 & 1.7705 \\ \mathrm{C} & 5.1714 & -2.3584 & -0.4798 \\ \mathrm{C} & 1.5806 & -1.4084 & 3.5229 \\ \mathrm{C} & 3.5923 & -3.5422 & 2.7007 \\ \mathrm{C} & 4.4141 & -1.3143 & 4.5927 \\ \mathrm{H} & 5.5889 & 1.5520 & 0.2997 \\ \mathrm{H} & 6.5465 & 0.5651 & -0.8159 \\ \mathrm{H} & 4.8146 & 0.7732 & -1.0860 \\ \mathrm{H} & 7.7189 & -1.2657 & 1.1262 \\ \mathrm{H} & 6.9949 & -0.3893 & 2.4890 \\ \mathrm{H} & 6.7669 & -2.1341 & 2.3373 \\ \mathrm{H} & 6.1138 & -2.4741 & -1.0287 \\ \mathrm{H} & 4.9644 & -3.2985 & 0.0401 \\ \mathrm{H} & 4.3757 & -2.2062 & -1.2141 \\ \mathrm{H} & 1.2857 & -2.1736 & 4.2506 \\ \mathrm{H} & 1.4263 & -0.4348 & 3.9951 \\ \mathrm{H} & 0.9143 & -1.4639 & 2.6604 \\ \mathrm{H} & 3.2451 & -4.1315 & 3.5579 \\ \mathrm{H} & 3.0193 & -3.8494 & 1.8207 \\ \mathrm{H} & 4.6403 & -3.8044 & 2.5205 \\ & 4.0794 & -1.9191 & 5.4441 \\ \mathrm{H} & 5.4763 & -1.5189 & 4.4278 \\ \mathrm{H} & & -0.2593 & 4.8745\end{array}$




\subsubsection{Pro-trans Epoxide Transition State Structure}

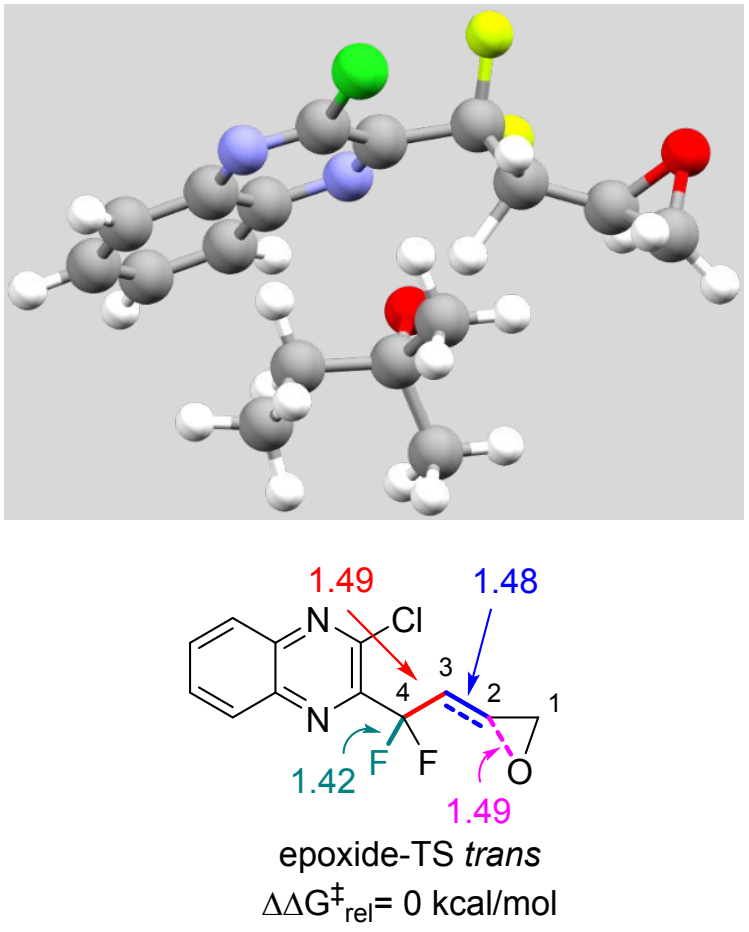

$\begin{array}{lrrr}\text { F } & 0.9790 & -0.2488 & -0.1256 \\ \text { C } & -0.4106 & 0.0548 & -0.1482 \\ \text { C } & -1.2139 & -0.5759 & 0.9328 \\ \text { C } & -1.7567 & -1.9190 & 0.6473 \\ \text { C } & -2.1251 & -2.8492 & 1.7176 \\ \text { O } & -0.9684 & -3.1513 & 0.9318 \\ \text { F } & -0.8174 & -0.3742 & -1.3917 \\ \text { C } & -0.5369 & 1.5846 & -0.1104 \\ \text { C } & -0.0961 & 2.3901 & 1.0063 \\ \text { Cl } & 0.8817 & 1.6947 & 2.3015 \\ \text { N } & -0.3385 & 3.6523 & 1.1185 \\ \text { C } & -1.0812 & 4.2367 & 0.1342 \\ \text { C } & -1.4461 & 5.5967 & 0.2347 \\ \text { C } & -2.1776 & 6.1843 & -0.7742 \\ \text { C } & -2.5595 & 5.4374 & -1.9125 \\ \text { C } & -2.2118 & 4.1095 & -2.0285 \\ \text { C } & -1.4719 & 3.4801 & -1.0012 \\ \text { N } & -1.1632 & 2.1598 & -1.1000 \\ \text { H } & -0.6571 & -0.5446 & 1.8680 \\ \text { H } & -2.2553 & 0.3323 & 1.1761 \\ \text { H } & -2.3020 & -2.0167 & -0.2877 \\ \text { H } & -2.9719 & -3.5271 & 1.5955 \\ \text { H } & -1.9254 & -2.5641 & 2.7511\end{array}$




$\begin{array}{rrrr}\mathrm{H} & -1.1429 & 6.1453 & 1.1190 \\ \mathrm{H} & -2.4679 & 7.2265 & -0.6959 \\ \mathrm{H} & -3.1396 & 5.9154 & -2.6947 \\ \mathrm{H} & -2.5058 & 3.5110 & -2.8824 \\ \mathrm{O} & -3.0160 & 1.3035 & 1.3086 \\ \mathrm{C} & -3.6315 & 1.5057 & 2.5435 \\ \mathrm{C} & -3.5577 & 3.0286 & 2.8368 \\ \mathrm{C} & -5.1033 & 1.0400 & 2.4586 \\ \mathrm{C} & -2.9157 & 0.7122 & 3.6611 \\ \mathrm{H} & -5.6479 & 1.1476 & 3.4054 \\ \mathrm{H} & -5.6199 & 1.6146 & 1.6839 \\ \mathrm{H} & -5.1239 & -0.0132 & 2.1638 \\ \mathrm{H} & -3.3923 & 0.8479 & 4.6382 \\ \mathrm{H} & -2.9312 & -0.3544 & 3.4220 \\ \mathrm{H} & -1.8700 & 1.0251 & 3.7332 \\ \mathrm{C} & -4.2306 & 3.5430 & 4.1159 \\ \mathrm{H} & -2.4964 & 3.2958 & 2.8463 \\ \mathrm{H} & -3.9890 & 3.5303 & 1.9635 \\ \mathrm{H} & -4.0959 & 4.6261 & 4.2164 \\ \mathrm{H} & -5.3072 & 3.3465 & 4.1171 \\ \mathrm{H} & -3.8081 & 3.0793 & 5.0124\end{array}$

\subsubsection{Pro-cis Epoxide Transition State Structure}

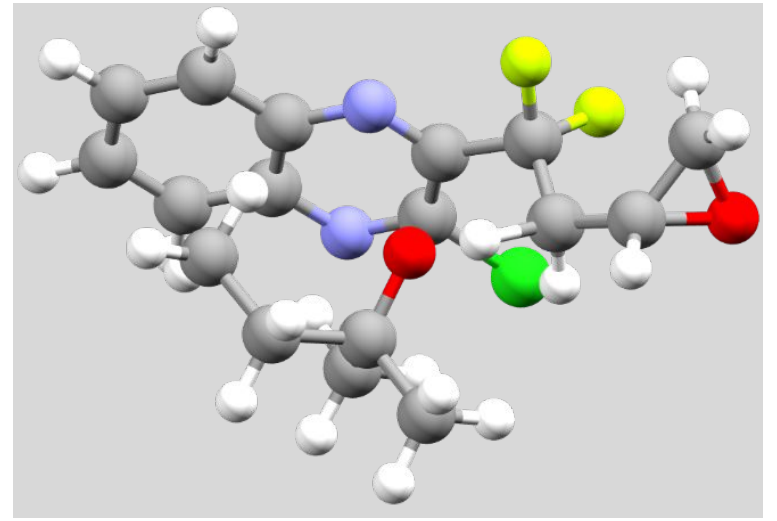

$$
\begin{aligned}
& \text { (1.43 } \\
& \text { epoxide-TS cis } \\
& \Delta \Delta \mathrm{G}_{\text {rel }}^{\ddagger}=0.4 \mathrm{kcal} / \mathrm{mol} \\
& \text { F } \quad \begin{array}{llll}
-0.8169 & -0.3829 & -1.3781
\end{array} \\
& \begin{array}{llll}
\text { C } & -0.0418 & 0.1455 & -0.3673
\end{array}
\end{aligned}
$$




\begin{tabular}{|c|c|c|c|}
\hline $\mathrm{C}$ & 1.3942 & 0.0718 & -0.6942 \\
\hline $\mathrm{C}$ & 1.9790 & -1.2299 & -1.1192 \\
\hline $\mathrm{C}$ & 1.2597 & -2.4995 & -1.2741 \\
\hline $\mathrm{O}$ & 2.1559 & -2.3469 & -0.1691 \\
\hline $\mathrm{F}$ & -0.4814 & -0.5980 & 0.7765 \\
\hline $\mathrm{C}$ & -0.4773 & 1.5981 & -0.1426 \\
\hline $\mathrm{C}$ & -0.0657 & 2.3938 & 0.9915 \\
\hline $\mathrm{Cl}$ & 0.8444 & 1.6851 & 2.3230 \\
\hline $\mathrm{N}$ & -0.3484 & 3.6505 & 1.1210 \\
\hline $\mathrm{C}$ & -1.0546 & 4.2456 & 0.1186 \\
\hline $\mathrm{C}$ & -1.3450 & 5.6273 & 0.1748 \\
\hline $\mathrm{C}$ & -2.0576 & 6.2147 & -0.8464 \\
\hline $\mathrm{C}$ & -2.5046 & 5.4478 & -1.9485 \\
\hline $\mathrm{C}$ & -2.2348 & 4.1005 & -2.0203 \\
\hline $\mathrm{C}$ & -1.4997 & 3.4737 & -0.9874 \\
\hline $\mathrm{N}$ & -1.2049 & 2.1515 & -1.0753 \\
\hline $\mathrm{H}$ & 1.5829 & 0.9 & -1.7343 \\
\hline $\mathrm{H}$ & 1.9850 & 0.5069 & 0.1128 \\
\hline $\mathrm{H}$ & 2.8710 & -1.11 & -1.7352 \\
\hline $\mathrm{H}$ & 1.5762 & -3.2 & -2.0420 \\
\hline $\mathrm{H}$ & 0.2069 & -2.5463 & -1.0132 \\
\hline $\mathrm{H}$ & -0.9857 & 6.1 & 1.0255 \\
\hline $\mathrm{H}$ & -2.2737 & 7.2771 & -0.8129 \\
\hline $\mathrm{H}$ & -3.0521 & 5.9334 & -2.7487 \\
\hline $\mathrm{H}$ & -2.5442 & 3.4938 & -2.8624 \\
\hline $\mathrm{O}$ & 1.7332 & 1.7165 & -2.7329 \\
\hline $\mathrm{C}$ & 2.6571 & 2.7398 & -2.5797 \\
\hline $\mathrm{C}$ & 2.4633 & 3.7551 & -3.7415 \\
\hline $\mathrm{C}$ & 2.4865 & 3.4697 & -1.2212 \\
\hline $\mathrm{C}$ & 4.1007 & 2.1836 & -2.65 \\
\hline $\mathrm{H}$ & 3.1819 & 4.3117 & -1.1153 \\
\hline $\mathrm{H}$ & 1.4695 & 3.8523 & -1.1211 \\
\hline $\mathrm{H}$ & 2.6653 & 2.7772 & -0.3951 \\
\hline $\mathrm{H}$ & 4.8639 & 2.9703 & -2.5797 \\
\hline $\mathrm{H}$ & 4.2603 & 1.4689 & -1.8399 \\
\hline $\mathrm{H}$ & 4.2318 & 1.6499 & -3.5997 \\
\hline $\mathrm{C}$ & 1.0388 & 4.3073 & -3.8397 \\
\hline $\mathrm{H}$ & 3.1878 & 4.5776 & -3.6476 \\
\hline $\mathrm{H}$ & 2.6989 & 3.2203 & -4.6693 \\
\hline $\mathrm{H}$ & 0.8978 & 4.8901 & -4.7575 \\
\hline $\mathrm{H}$ & 0.3340 & 3.4732 & -3.8347 \\
\hline $\mathrm{H}$ & 0.7915 & 4.9595 & -2.9962 \\
\hline
\end{tabular}


1.2. Energy Calculations

\begin{tabular}{ccccccc}
\hline Entry & Transition State & $\begin{array}{c}\text { Temp } \\
\left({ }^{\circ} \mathrm{C}\right)\end{array}$ & $\begin{array}{c}\Delta \mathrm{G}_{\mathrm{gas}}^{\ddagger} \\
(\text { hartree })^{\mathrm{a}}\end{array}$ & $\begin{array}{c}\Delta \mathrm{E}_{\text {gas }}^{\ddagger} \\
(\text { hartree })^{\mathrm{a}}\end{array}$ & $\begin{array}{c}\Delta \mathrm{G}_{\text {solv }}^{\ddagger}(\text { corrected }) \\
(\text { hartree })^{\mathrm{b}}\end{array}$ & $\begin{array}{c}\Delta \Delta \mathrm{G}_{\text {rel }}^{\ddagger} \\
(\mathrm{kcal} / \mathrm{mol})^{\mathrm{c}}\end{array}$ \\
\hline 1 & Pro-trans Carbonate & -10 & -2369.40342 & -2369.77778 & -2369.482435 & 0.00 \\
2 & Pro-cis Carbonate & -10 & -2369.39725 & -2369.774173 & -2369.475494 & 4.36 \\
3 & Pro-trans Epoxide & -10 & -1579.75247 & -1580.05667 & $-1579.826943^{\mathrm{d}}$ & 0.00 \\
4 & Pro-cis Epoxide & -10 & -1579.76224 & -1580.054363 & $-1579.826248^{\mathrm{e}}$ & 0.44
\end{tabular}

(a) Calculated at B3LYP-D3/6-311G**++ level of theory. (b) Calculated at B3LYP-D3/6-311G**++/SM8 level of theory and corrected by adding ( $\left.\Delta \mathrm{G}^{\ddagger}{ }_{\mathrm{gas}}-\Delta \mathrm{E}_{\mathrm{gas}}^{\ddagger}\right)$ to the uncorrected value of $\Delta \mathrm{G}_{\text {solv }}^{\ddagger}$. (c) Calculated by subtracting corrected $\Delta \mathrm{G}_{\text {solv }}^{\ddagger}($ cis $)-\Delta \mathrm{G}_{\text {solv }}^{\star}$ (trans) for each isomer pair at the same temperature and converting to $\mathrm{kcal} / \mathrm{mol}$ from hartree ( 1 hartree $=627.509 \mathrm{kcal} / \mathrm{mol})$. (d) THF used as the solvent in the calculation. (e) Benzene used as the solvent in the calculation.

\subsection{Discussion of Negative Hyperconjugation in the TS as a Stabilizing Factor}

The transition states identified for both substrates show significant E2 character, with shortening of the $\mathrm{C} 2-\mathrm{C} 3$ bonds, hybridization of the carbon atoms in the forming olefin, and the lengthening of the carbonate $\mathrm{C} 1-\mathrm{O}$ bond. The degree of hybridization in the carbon being deprotonated suggests the transition state is early, and that the elimination itself is asynchronous, with significant negative charge building up on the deprotonated carbon. Additionally, the neighboring $\mathrm{C} 3-\mathrm{C} 4$ bond is shortened, evidence of negative hyperconjugation between the developing anion and the $\sigma^{*}$ of the antiperiplanar C-F bond. This stabilizing interaction is observed in both the cis and trans transition states for each substrate, and the bond lengths suggests a similar amount of partial double bond character along the forming olefin and $\mathrm{C} 3-\mathrm{C} 4$ bond.

Observation of the HOMO suggests that there is significant negative hyperconjugation of the developing anionic charge and the $\sigma^{*}$ orbital of the antiperiplanar $\mathrm{C}-\mathrm{F}$ bond on the neighboring carbon (Figure S1). The transition states located for the epoxide elimination exhibit similar properties regarding the asynchronous nature of the transition state. It is still concerted, as the partial olefin character of the $\mathrm{C} 2-\mathrm{C} 3$ bond and increasing length of the $\mathrm{C} 1-\mathrm{O}$ bond is still observed in the epoxide substrates. The epoxide substrates also exhibit the negative hyperconjugation observed in the carbonate transition states. However, in the epoxide case the HOMO appears to be more polarized in the direction of the CF2 group on $\mathrm{C} 4$. It is speculated that the decreased orbital overlap with the epoxide due to the geometry of the group is the main cause of increased orbital contribution toward the CF2 group. This hyperconjugation also explains the C-F elimination observed as an impurity in these eliminations. 

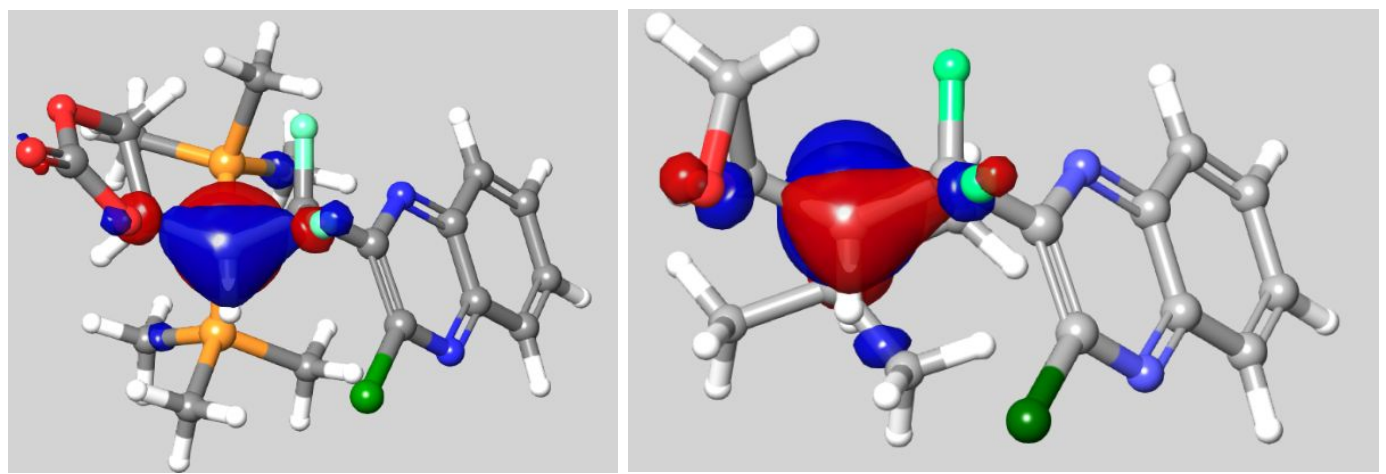

Figure S1. Left: HOMO of carbonate-TS cis, exhibiting nearly symmetrical delocalization along both $\mathrm{C} 2-\mathrm{C} 3$, and $\mathrm{C} 3-\mathrm{C} 4$ bonds. Right: $\mathrm{HOMO}$ of epoxide-TS cis, exhibiting increased delocalization along the $\mathrm{C} 3-\mathrm{C} 4$ bond toward the fluorine atoms. 


\section{NMR Spectra}
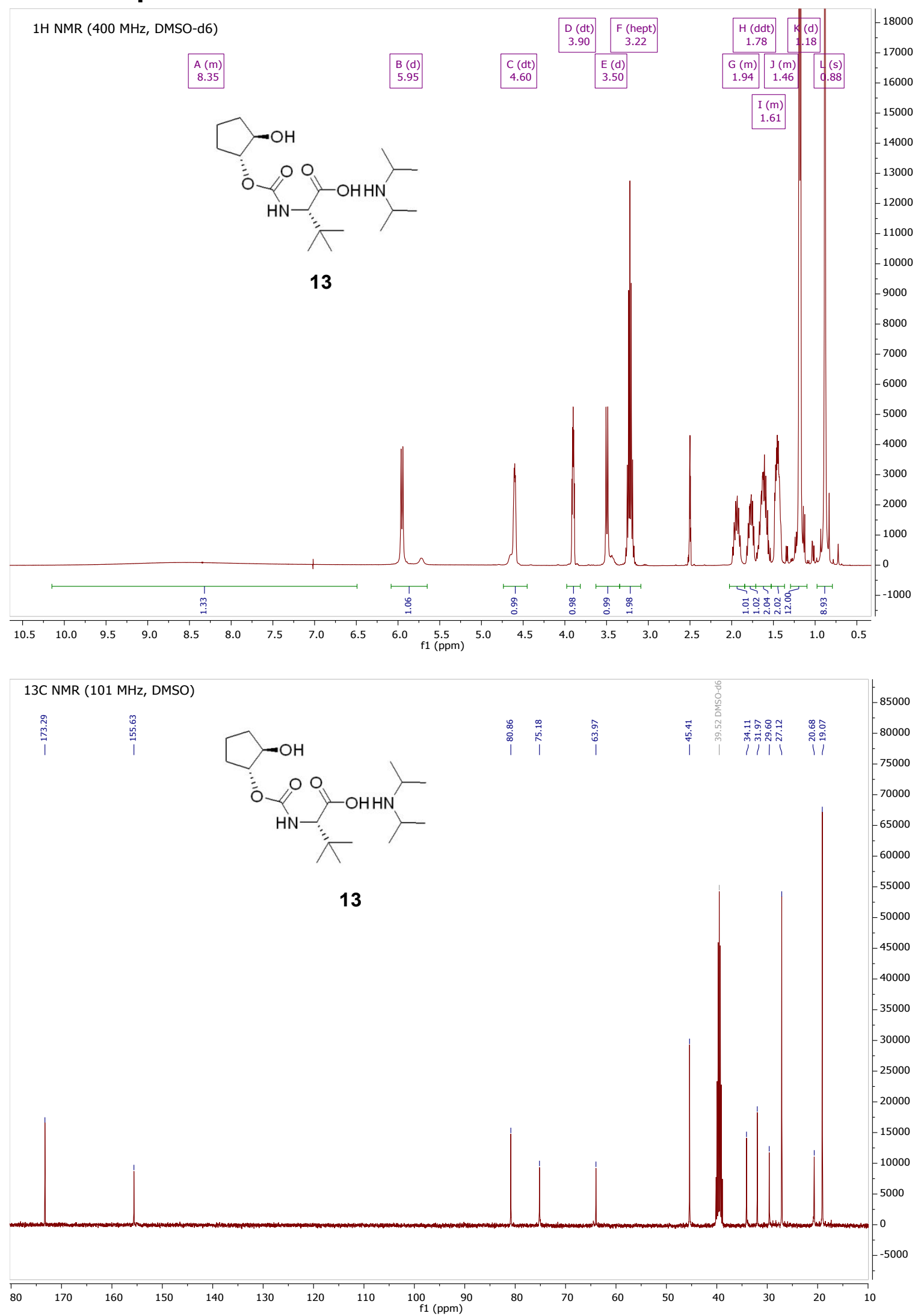
1H NMR (400 MHz, DMSO-d6)

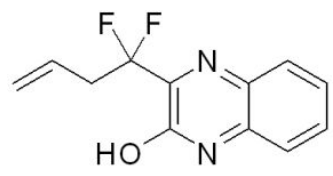

43
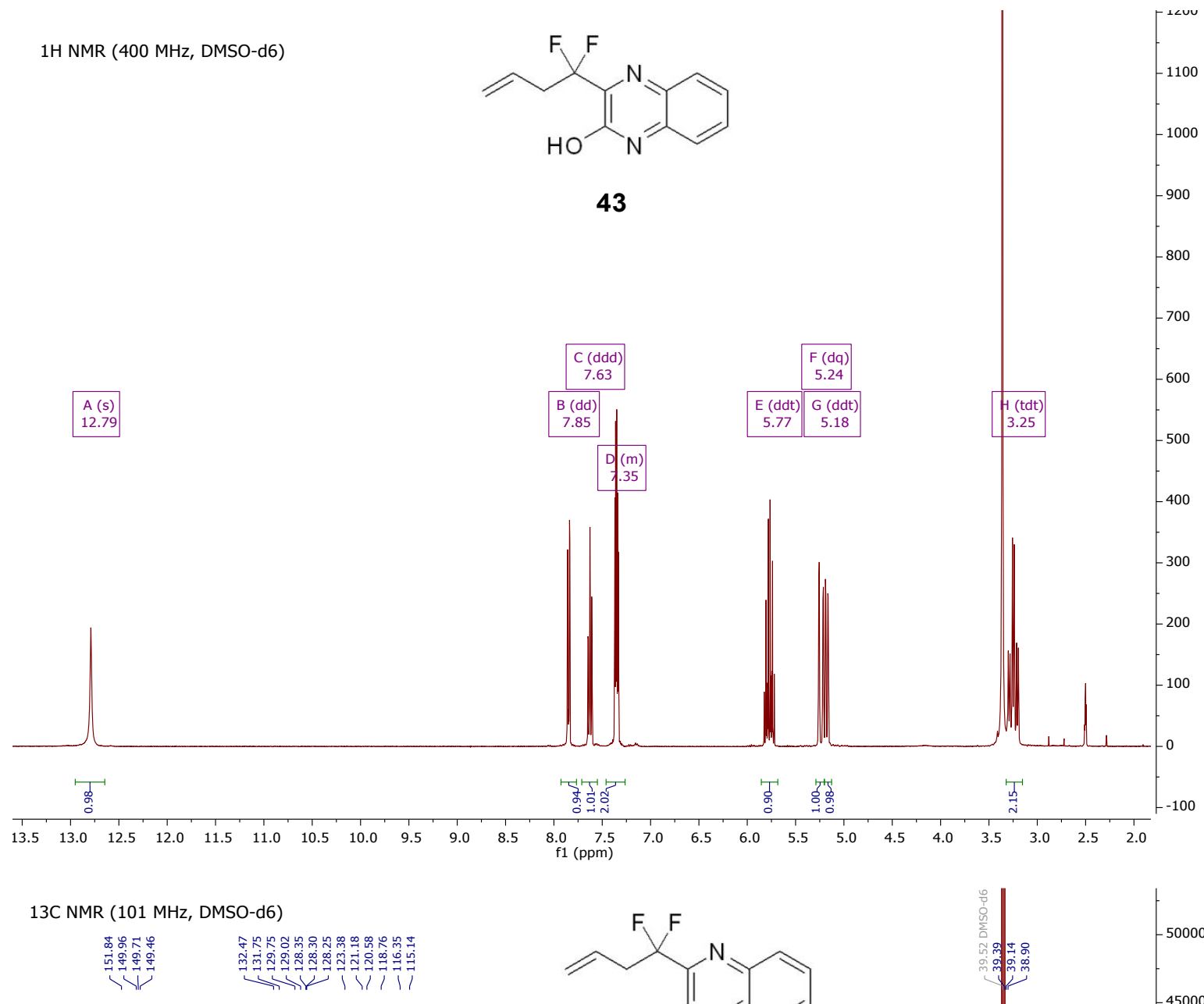

900

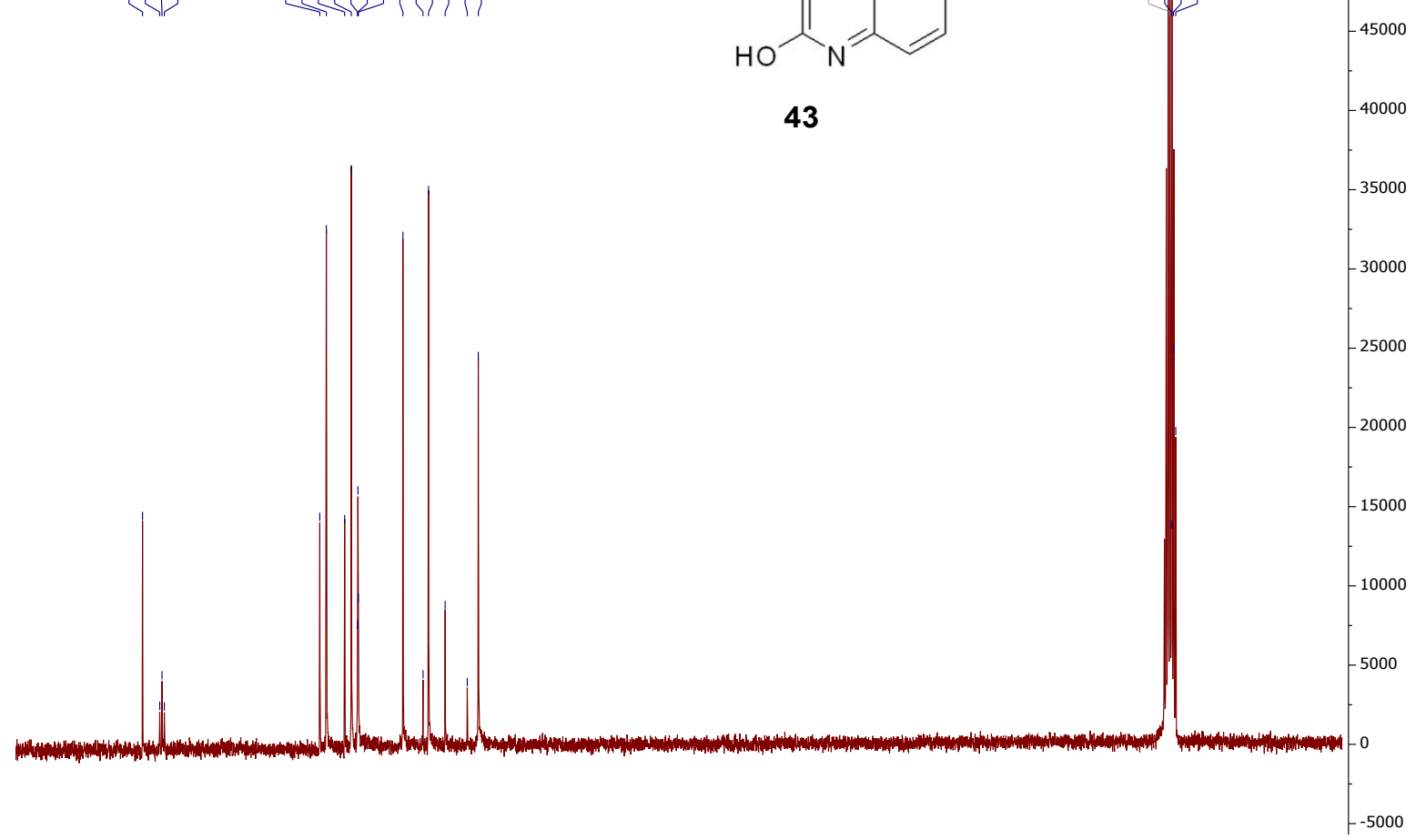

\begin{tabular}{llllllllllllllllllllllllllllllll}
\hline 65 & 160 & 155 & 150 & 145 & 140 & 135 & 130 & 125 & 120 & 115 & 110 & 105 & 100 & 95 & 90 & 85 & 80 & 75 & 70 & 65 & 60 & 55 & 50 & 45 & 40 & 35 & 30 & 25
\end{tabular} 
1H NMR (400 MHz, Chloroform-d)
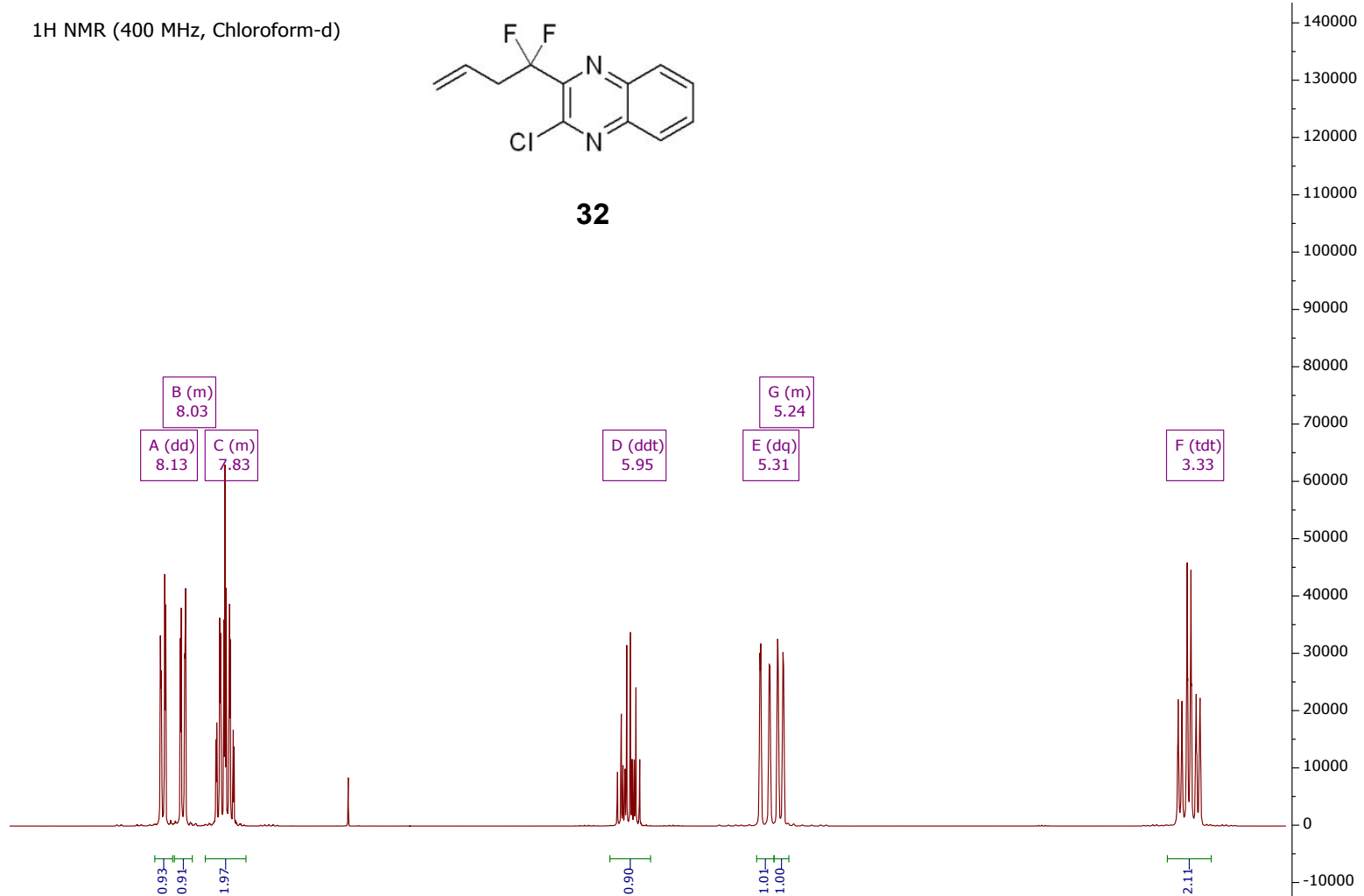

\begin{tabular}{c}
\hline G (m) \\
5.24 \\
\hline$(\mathrm{dq})$ \\
\hline
\end{tabular}

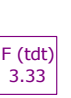

60000

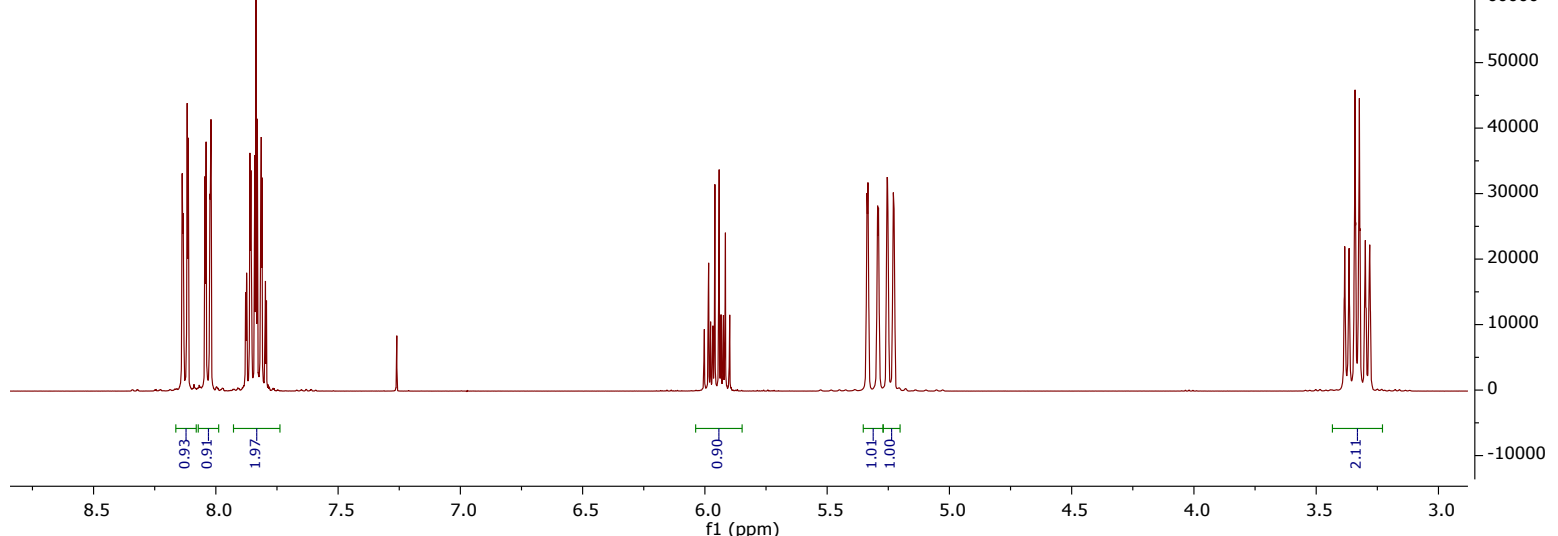

13C्र NMR (101 MHzz

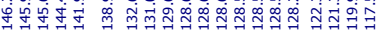

计, 等,
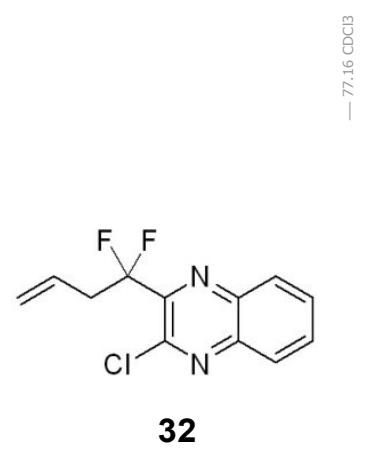

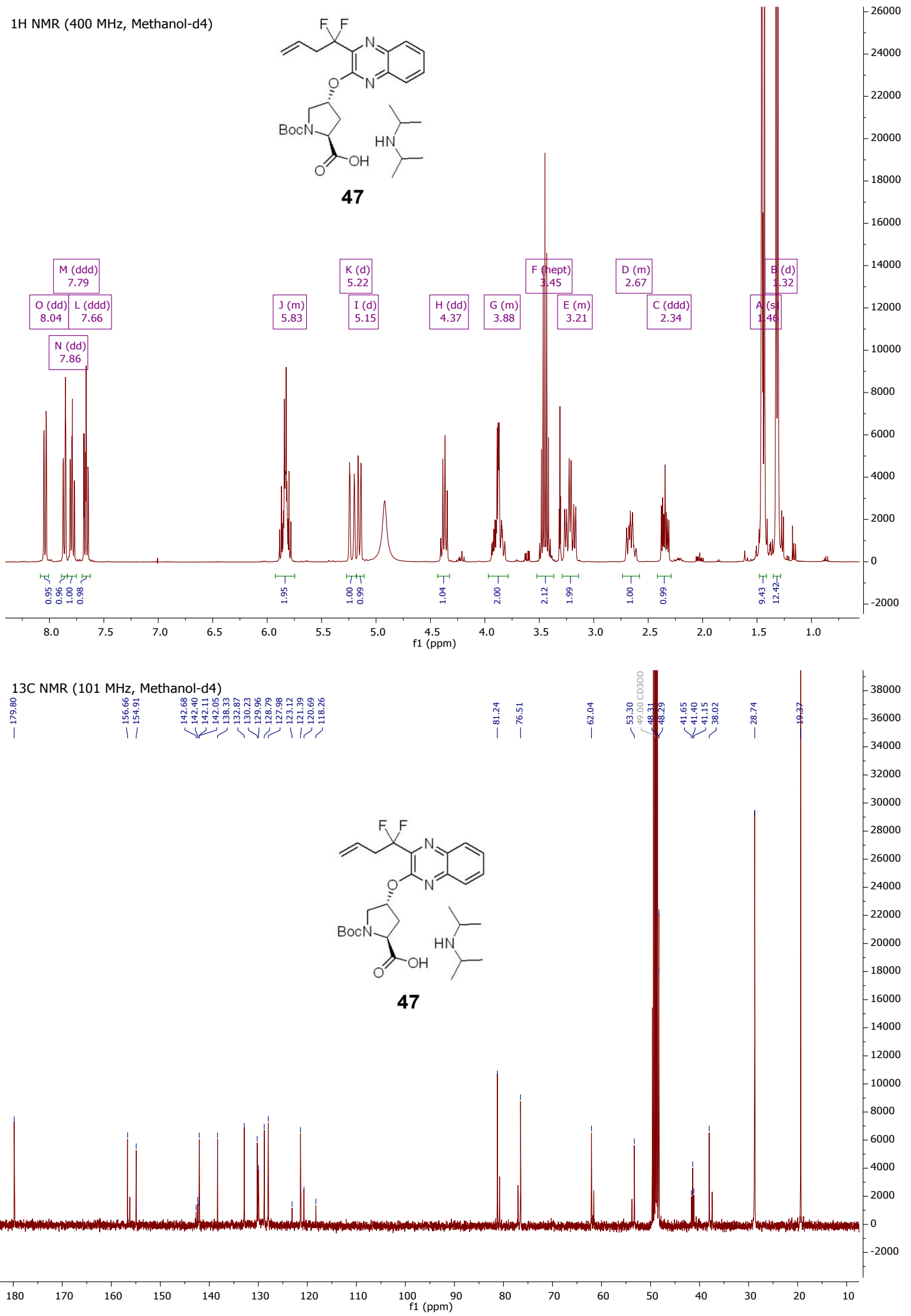

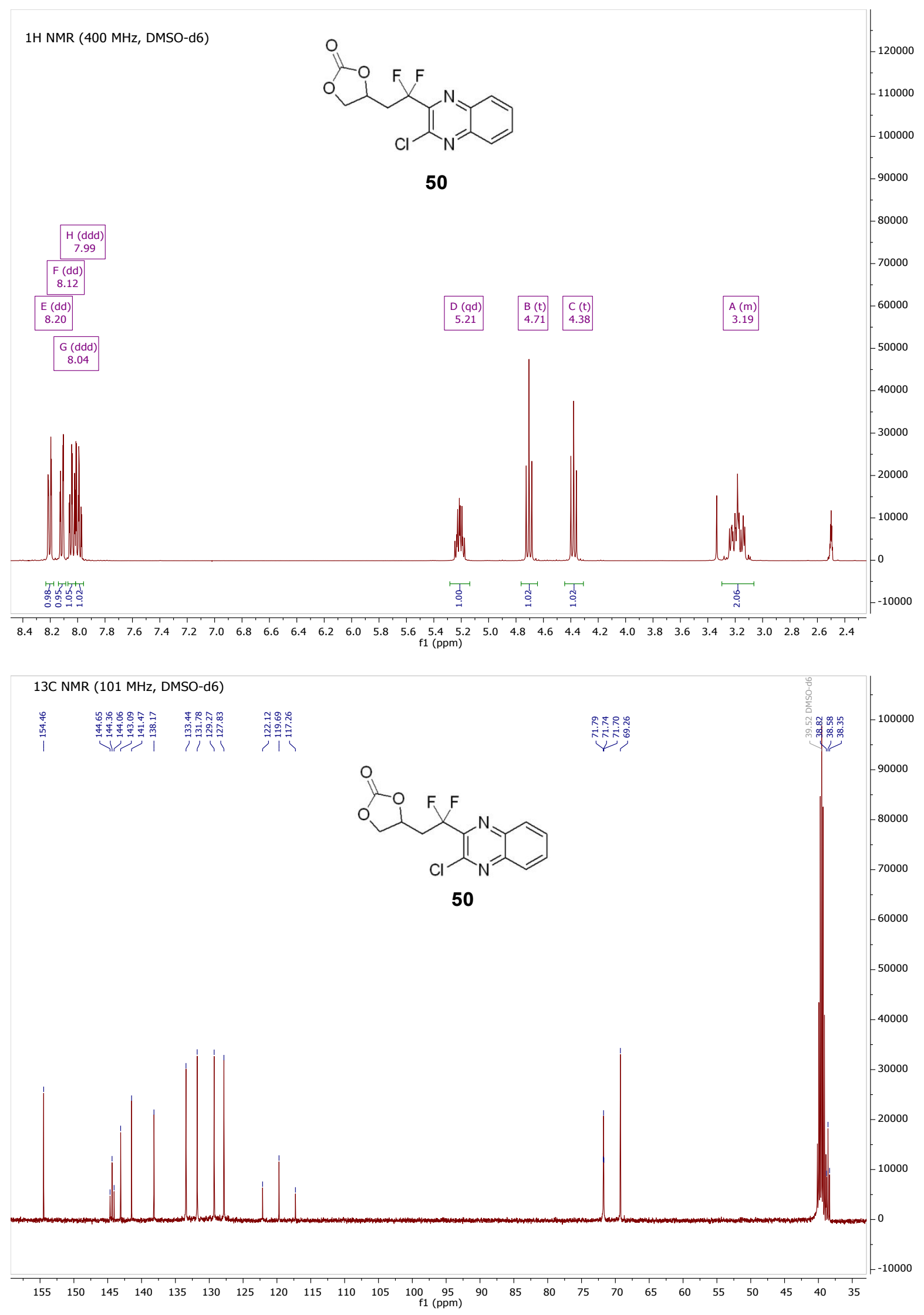

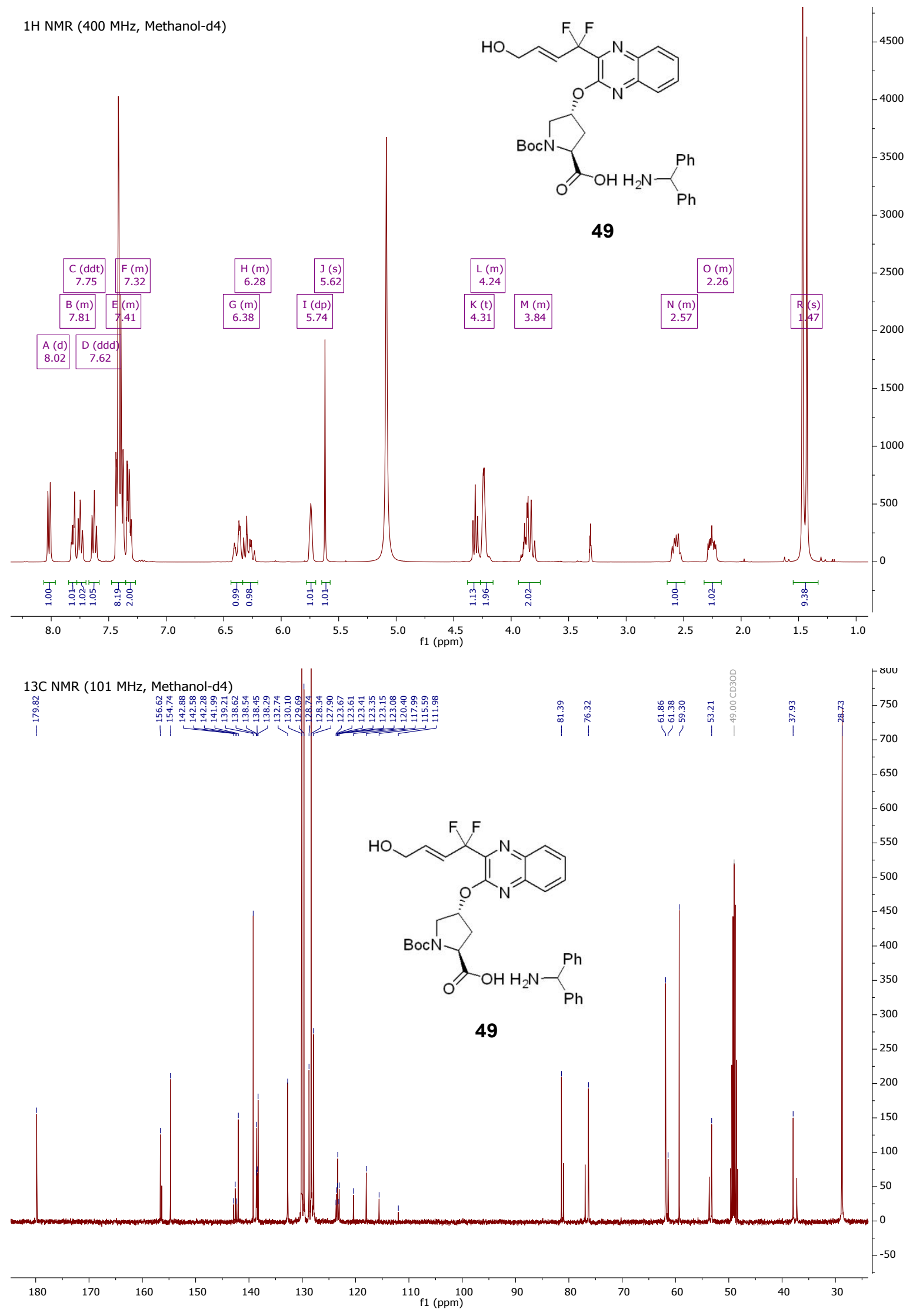
1H NMR (400 MHz, Methanol-d4)

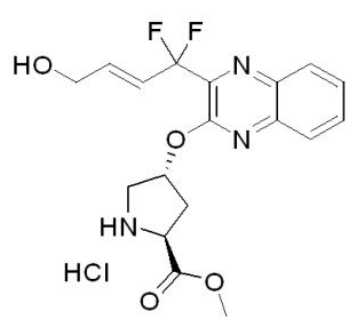

31
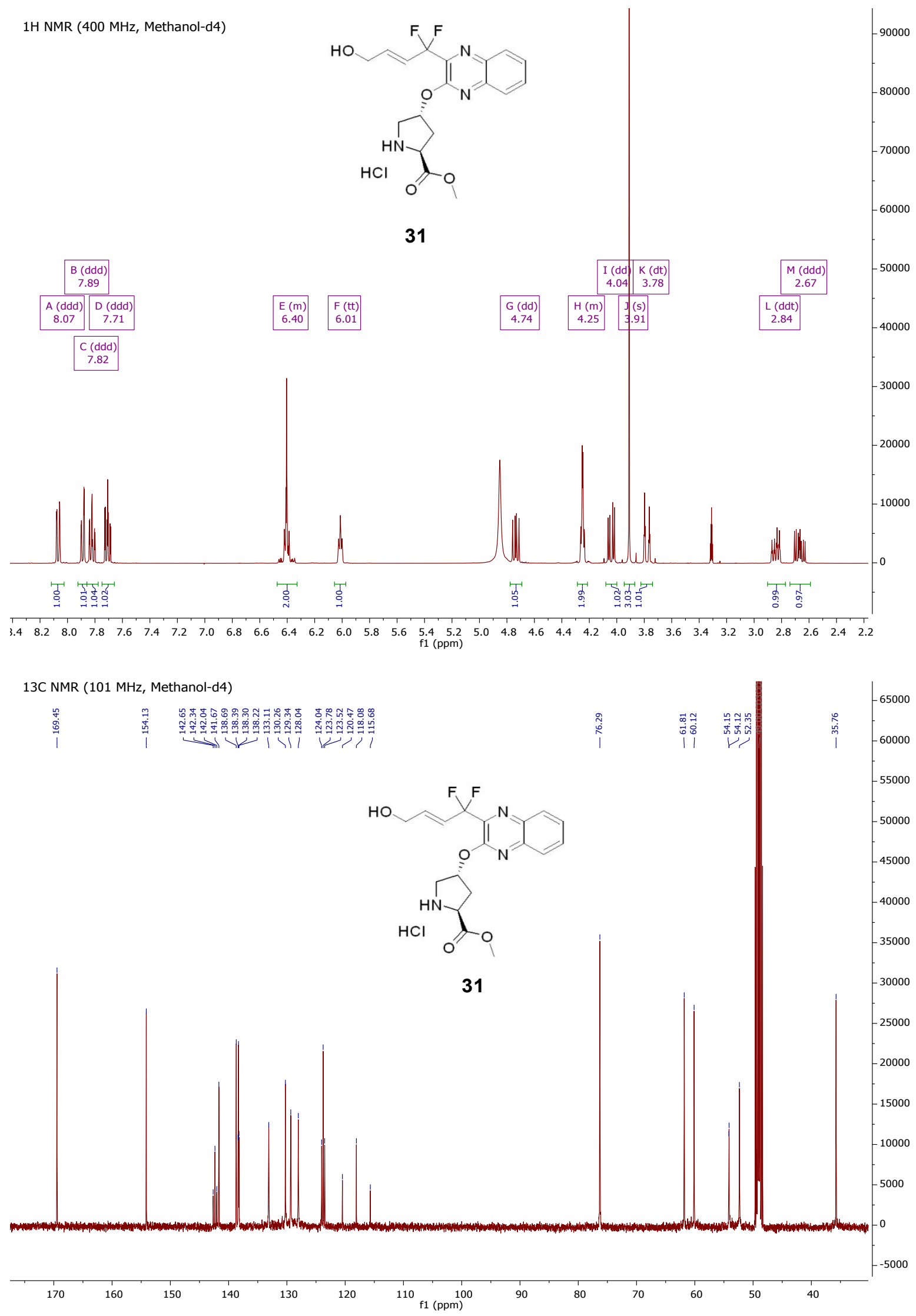

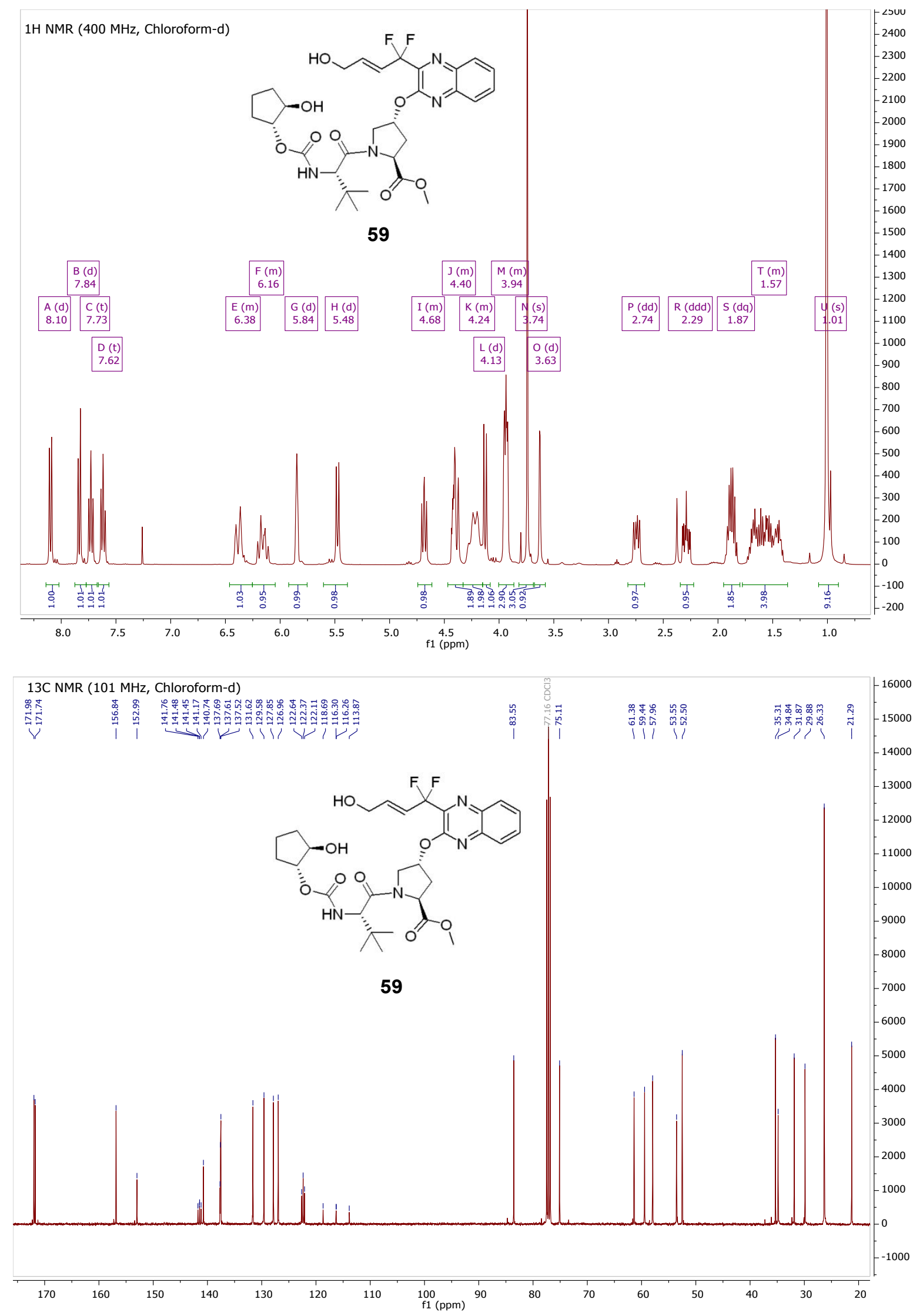


\section{Single Crystal X-ray Structure Details}

\subsection{Macrocycle $3 p$-Xylene Solvate Single Crystal Structure}

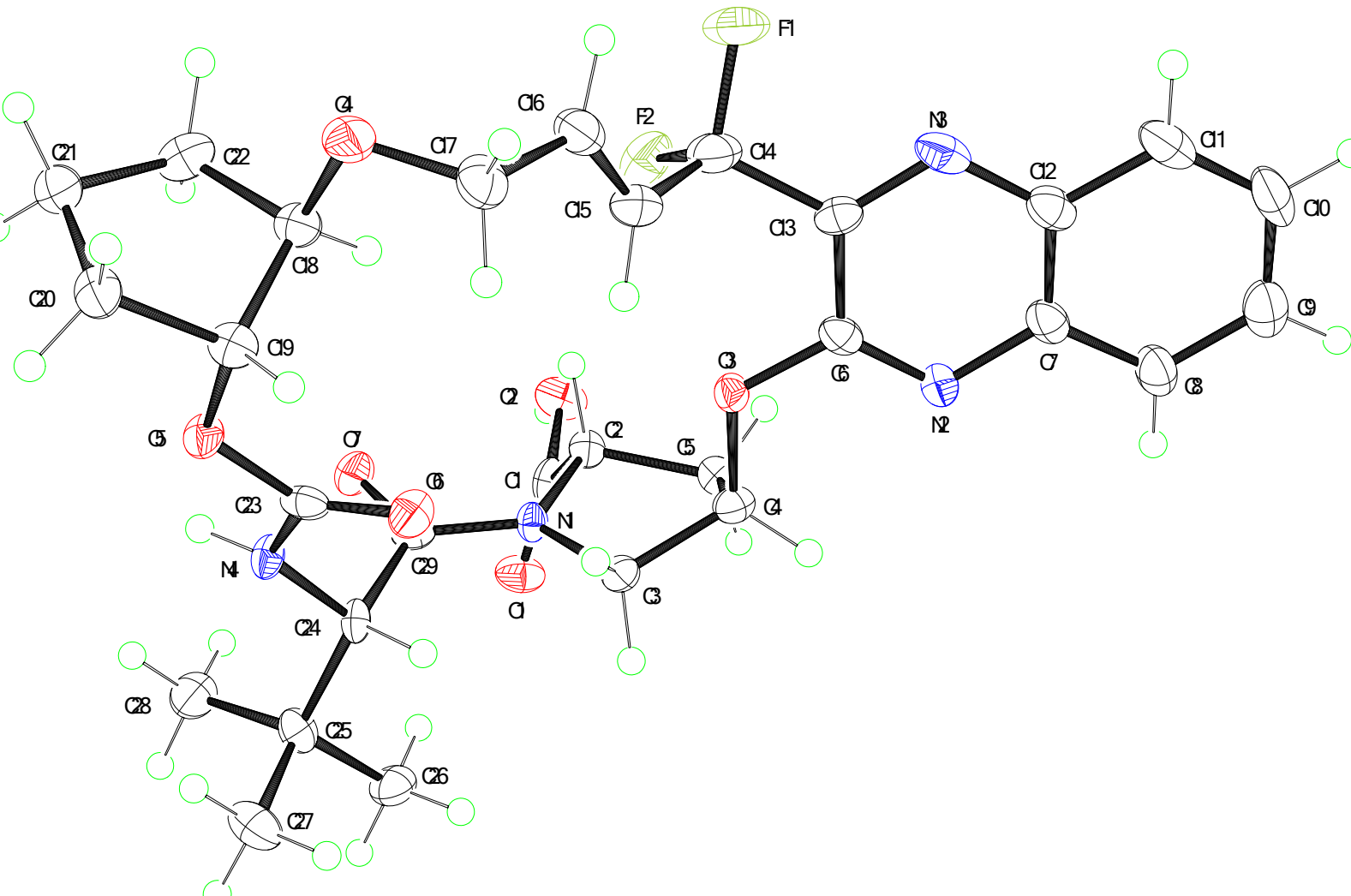

Figure S2. ORTEP diagram for Macrocycle 3 p-Xylene Solvate structure. The solvent molecule and partially occupied water oxygen atom were omitted for clarity.

Structure refinement details: The xylene solvent molecule was disordered across a symmetry element and was modeled thus, with geometric and anisotropic displacement constraints applied. The partially occupied water molecule was allowed to refine with a final occupancy of $\sim 49 \%$.

\subsection{Macrocycle 3 Toluene Solvate Single Crystal Structure}

Structure refinement details: The toluene solvent molecule was disordered across a symmetry element and was modeled thus, with geometric and anisotropic displacement constraints applied. The partially occupied water molecule was allowed to refine with a final occupancy of $\sim 39 \%$. 


\subsection{Macrocycle 3 Acetonitrile Solvate Single Crystal Structure}

Structure refinement details: Thermal disorder in select atoms of the acetonitrile solvent molecules could not be treated effectively by constraints. 